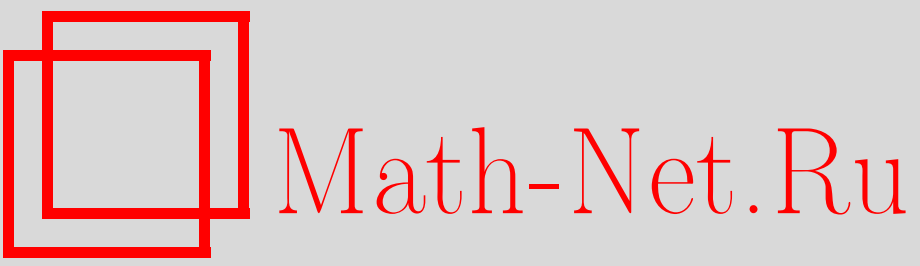

Л. В. Богачев, Е. Б. Яровая, О моментах ветвящегося случайного блуждания в случайной среде, УМН, 2000, том 55, выпуск 5, 173-174

DOI: https://doi.org/10.4213/rm325

Использование Общероссийского математического портала Math-Net.Ru подразумевает, что вы прочитали и согласны с пользовательским соглашением

http://www.mathnet.ru/rus/agreement

Параметры загрузки:

IP: 35.173 .219 .12

26 апреля 2023 г., 17:53:21 


\title{
О МОМЕНТАХ ВЕТВЯЩЕГОСЯ СЛУЧАЙНОГО БЛУЖДАНИЯ В СЛУЧАЙНОЙ СРЕДЕ
}

\author{
Л.В. БОГАЧЕВ, Е. Б. ЯРОВАЯ
}

1. В работе рассматривается ветвящееся случайное блуждание с непрерывным временем на $\mathbb{Z}^{d}(d \geqslant 1)$. Ветвящаяся среда (т.е. интенсивности рождения и гибели частиц в узлах решетки) является случайной и задается случайными полями $\xi_{2}(\cdot) \geqslant 0, \xi_{0}(\cdot) \geqslant 0$ на $\mathbb{Z}^{d}$, определенными на некотором вероятностном пространстве $(\Omega, \mathscr{F}, \mathrm{P})$. "Диффузия" частиц происходит по закону простого симметричного случайного блуждания с интенсивностью скачков $\varkappa>0$. Таким образом, при фиксированной реализации $\omega \in \Omega$ случайной среды эволюция ансамбля частиц описывается однородным марковским процессом с переходами следующего вида: частица, находящаяся в точке $x$, за время $h \rightarrow 0$ независимо от остальных частиц с вероятностью $(2 d)^{-1} \varkappa h$ перепрыгивает в соседнюю точку $x^{\prime}\left(\left|x^{\prime}-x\right|=1\right)$, с вероятностью $\xi_{2}(x) h$ делится на две частицы, а с вероятностью $\xi_{0}(x) h$ погибает. (Формальное построение такого процесса см., например, в $[1 ;$ гл. $\mathrm{V}, \S 3]$.

2. Пусть $\mu_{t}(y)$ - число частиц в точке $y \in \mathbb{Z}^{d}$ в момент времени $t, \mu_{t}:=\sum_{y} \mu_{t}(y)$. Будем считать, что при $t=0$ имеется единственная частица, расположенная в точке $x \in \mathbb{Z}^{d}$, т.е. $\mu_{0}=1$, $\mu_{0}(y)=\delta_{x}(y)$ (здесь и далее $\delta_{x}(y)$ - символ Кронекера). Обозначим через $\mathrm{E}_{x}^{(\omega)}$ математическое ожидание относительно распределения процесса в "замороженной" случайной среде. Хорошо известно (см. [2], [3]), что производящие функции $F(z ; t, x, y):=\mathrm{E}_{x}^{(\omega)}\left[e^{-z \mu_{t}(y)}\right], F(z ; t, x):=$ $\mathrm{E}_{x}^{(\omega)}\left[e^{-z \mu_{t}}\right](z \geqslant 0)$ удовлетворяют обратному уравнению Колмогорова

$$
\partial_{t} F=\varkappa \Delta F+\left[\xi_{2}(x) F-\xi_{0}(x)\right] \cdot(F-1)
$$

с начальными условиями

$$
F(z ; 0, x, y)=e^{-z \delta_{y}(x)}, \quad F(z ; 0, x)=e^{-z},
$$

где $\partial_{t}:=\partial / \partial t$, а $\Delta-$ разностный лапласиан, действуюший на функции $\psi: \mathbb{Z}^{d} \rightarrow \mathbb{R}$ по формуле $\Delta \psi(x)=(2 d)^{-1} \sum_{\left|x^{\prime}-x\right|=1} \psi\left(x^{\prime}\right)-\psi(x)$.

Рассмотрим моменты $m_{n}(t, x, y):=\mathrm{E}_{x}^{(\omega)}\left[\mu_{t}^{n}(y)\right], m_{n}(t, x):=\mathrm{E}_{x}^{(\omega)}\left[\mu_{t}^{n}\right](n \in \mathbb{N})$. Дифференцируя соотношения (1), (2) по $z$ при $z=0$, можно показать (см. [4]-[6]), что функции $\left\{m_{n}(t, x, y)\right\}$, $\left\{m_{n}(t, x)\right\}$ удовлетворяют цепочке линейных (неоднородных) уравнений

$$
\partial_{t} m_{n}=\varkappa \Delta m_{n}+\xi(x) m_{n}+\xi_{2}(x) g_{n}\left[m_{1}, \ldots, m_{n-1}\right], \quad n=1,2, \ldots,
$$

с начальными условиями

$$
m_{n}(0, x, y)=\delta_{y}(x), \quad m_{n}(0, x) \equiv 1, \quad n=1,2, \ldots,
$$

где $\xi(x):=\xi_{2}(x)-\xi_{0}(x), g_{1} \equiv 0$, а при $n \geqslant 2$

$$
g_{n}\left[m_{1}, \ldots, m_{n-1}\right]:=\sum_{i=1}^{n-1} \frac{n !}{i !(n-i) !} m_{i} m_{n-i} .
$$

3. Пусть $\xi(\cdot), \eta(t, \cdot) \geqslant 0, v_{0}(\cdot) \geqslant 0$-случайные поля на $\mathbb{Z}^{d}$, определенные на вероятностном пространстве $(\Omega, \mathscr{F}, \mathrm{P})$. Рассмотрим неоднородную задачу Коши

$$
\partial_{t} v(t, x)=\varkappa \Delta v(t, x)+\xi(x) v(t, x)+\eta(t, x), \quad v(0, x)=v_{0}(x) .
$$

Положим $\ln _{+} x:=\max \{\ln x, 1\}$ и обозначим через $\Phi$ класс функций $\varphi: \mathbb{Z}^{d} \rightarrow \mathbb{R}_{+}$, удовлетворяющих условию $\lim \sup _{|x| \rightarrow \infty}(|x| \ln |x|)^{-1} \ln _{+} \varphi(x)<1$.

Работа выполнена при частичной финансовой поддержке РФФИ (грант № 99-01-00298) и DFG (грант № 436 RUS 113/534). 
Теорема 1. Предположим, что случайнъе величины $\xi(x), x \in \mathbb{Z}^{d}$, независимы и одинаково распределены, и пусть справедливо условие

$$
\left\langle\left(\frac{\xi^{+}(0)}{\ln _{+} \xi(0)}\right)^{d}\right\rangle<\infty
$$

где $\xi^{+}:=\max (\xi, 0)$. Пусть Р-n.н. выполнень следующие условия: а) $v_{0}(\cdot) \in \Phi$; б) при каждом $x$ функция $\eta(t, x)$ непрерывна по $t$; в) $Y(t, \cdot):=\max _{0 \leqslant s \leqslant t} \eta(s, \cdot) \in \Phi$ при каждом $t$. Тогда с вероятностью 1 задача (6) имеет единственное неотричательное решение

$$
v(t, x)=\mathrm{E}_{x}\left[e^{\int_{0}^{t} \xi\left(x_{s}\right) d s} v_{0}\left(x_{t}\right)\right]+\mathrm{E}_{x}\left[\int_{0}^{t} e^{\int_{0}^{s} \xi\left(x_{u}\right) d u} \eta\left(t-s, x_{s}\right) d s\right]
$$

где $x_{t}$ - случайное блуждание на $\mathbb{Z}^{d}$ с генератором $\varkappa \Delta$, а $\mathrm{E}_{x}$ - соответствующее математическое ожидание при условии $x_{0}=x$.

Отметим, что в частном случае $\eta \equiv 0$ представление (8) сводится к известной формуле Фейнмана-Каца для уравнения Андерсона со случайным потенциалом (см. [3; теорема 2.1]).

4. Применяя теорему 1 к задаче Коши (3), (4), получаем следуюший результат.

Теорема 2. Предположим, что пары $\left(\xi_{2}(x), \xi_{0}(x)\right), x \in \mathbb{Z}^{d}$, независимы и одинаково распределень. Пусть выполнено условие (7) и, кроме того,

$$
\left\langle\left(\frac{\ln _{+} \xi_{2}(0)}{\ln _{+} \ln _{+} \xi_{2}(0)}\right)^{d}\right\rangle<\infty
$$

Тогда при каждом $n \in \mathbb{N}$ с вероятностью 1 имеют место представления

$$
\begin{aligned}
m_{n}(t, x, y) & =\mathrm{E}_{x}\left[e^{\int_{0}^{t} \xi\left(x_{s}\right) d s} \delta_{y}\left(x_{t}\right)\right]+\mathrm{E}_{x}\left[\int_{0}^{t} e^{\int_{0}^{s} \xi\left(x_{u}\right) d u} \xi_{2}\left(x_{s}\right) g_{n}\left(t-s, x_{s}, y\right) d s\right], \\
m_{n}(t, x) & =\mathrm{E}_{x}\left[e^{\int_{0}^{t} \xi\left(x_{s}\right) d s}\right]+\mathrm{E}_{x}\left[\int_{0}^{t} e^{\int_{0}^{s} \xi\left(x_{u}\right) d u} \xi_{2}\left(x_{s}\right) g_{n}\left(t-s, x_{s}\right) d s\right],
\end{aligned}
$$

где $g_{n}=g_{n}\left[m_{1}, \ldots, m_{n-1}\right]-$ функция, определенная в (5).

5. В следующей теореме для полных моментов $\left\langle m_{n}^{p}(t, x, y)\right\rangle,\left\langle m_{n}^{p}(t, x)\right\rangle$ находится асимптотика при $t \rightarrow \infty$ в предположении, что распределение $\xi$ имеет вейбулловский "хвост" на $+\infty$. (Аналогичньй результат для случая чистого размножения $\left(\xi_{0} \equiv 0\right)$ получен в работах [4], [5].)

ТЕОрема 3. Пусть выполнены условия теоремы 2. Предположим, что

$$
\lim _{z \rightarrow+\infty} z^{-\alpha} \ln \mathrm{P}\{\xi(0)>z\}=-c
$$

при некоторых $\alpha>1, c>0$. Тогда при всех $n, p \in \mathbb{N}$

$$
\lim _{t \rightarrow \infty} t^{-\alpha^{\prime}} \ln \left\langle m_{n}^{p}\right\rangle=\gamma(\alpha, c)(n p)^{\alpha^{\prime}},
$$

әде $\alpha^{\prime}:=\alpha /(\alpha-1), \gamma(\alpha, c):=(\alpha-1) \alpha^{-\alpha^{\prime}} c^{-1 /(\alpha-1)}$.

Отметим, что условие (7), накладываемое в теореме 2, автоматически выполнено в силу (9).

Авторы выражают благодарность С. Альбеверио и С. А. Молчанову за полезные дискуссии.

\section{СПИСОК ЛИТЕРАТУРЫ}

[1] Гихман И. И., Скороход А. В. Теория случайных процессов. Т. II. М.: Наука, 1973. [2] Скороход А. В. // Теория вероятн. и ее примен. 1964. Т. 9. С. 492-497. [3] Gärtner J., Molchanov S. A. // Comm. Math. Phys. 1990. V. 132. P. 613-655. [4] Яровая E. Б. // Вестн. МГУ. Сер. 1. Матем. Mex. 1990. № 4. C. 79-82. [5] Molchanov S. A. // IMA Vol. Math. Appl. 1996. V. 77. P. 81-109. [6] Albeverio S., Bogachev L. V.// Positivity. 2000. V. 4. P. 41-100.

Московский государственный университет им. М.В. Ломоносова; ГНИЦ профилактической медицины Минздрава РФ, г. Москва E-mail: lvb@mech.math.msu.su, yarov@cardpl.msk.ru
Принято редколлегией 07.09.2000 